\title{
Design of a New Charging Device based on the Slider Crank Mechanism Jinliang LI
}

\author{
Zibo Vocational Institute Industrial Engineering and Operations Department, 255314, China \\ email:lj|522@163.com
}

Keywords: new type pump; optimization design; overload protection

\begin{abstract}
The new kind of pedal pump that handling easy, safe and reliable, versatile was designed, its working principle was detailed shown. on the condition of meeting the design requirements and the actual, its' mechanism was the developed and optimized by the type synthesis of mechanisms and virtual prototype technology. The equipment could realize pressure adjustment, cut down the cost, and increase the charging efficiency.
\end{abstract}

\section{Introduction}

With the improvement of urbanization level, the number of vehicle grow rapidly, which the number of automobile, motorcycle, bicycle, electric cars were near the top in the world. These vehicles used pneumatic rubber tire, when traveling leakage, pneumatic has put people to great inconvenience. To this there were different solutions in different countries and regions for users. In Europe, the pump was carried on the bike fixed position, and using advanced self inflating tire technology. Most of the vehicles were inflated in the repair which was distributed irregular, wasting time and strength. Especially the problems of pressure control and overload protection were need to solve. In this paper, a pedal type inflating mechanism was designed, which has the advantages of large bearing capacity, inflation adjusted, overload protection. This equipment makes its work more stable and safety, small volume, easy to use.

\section{Mechanism Design}

New type pump used planar linkage mechanism depending on foot reinforcement, which was compact and convenient and easy to carry storage, overload protection device was set up to prevent the inflation pressure high. The new type pump could be used for bicycle, electric vehicle, the private vehicle tire inflation. The executive device of inflation mechanism was piston, which moving back-and-forth steadily. Though analysing, its mechanism was designed as single degree of freedom motion mechanism, then next step put its emphasis on the choice of planar linkage mechanism and the whole process of structural design. Selecting different pair combinations, 3 Inflatable mechanism schemes were selected. Respectively, advantages and disadvantages of each scheme of mechanism are analyzed in detail, and finally the slider crank mechanism as the design plan inflation mechanism, as shown in Figure 1 and Figure 2.

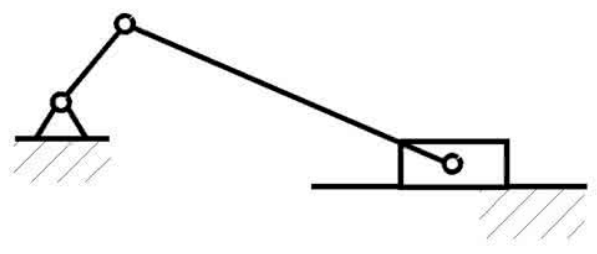

Figure 1 slider crank mechanism 


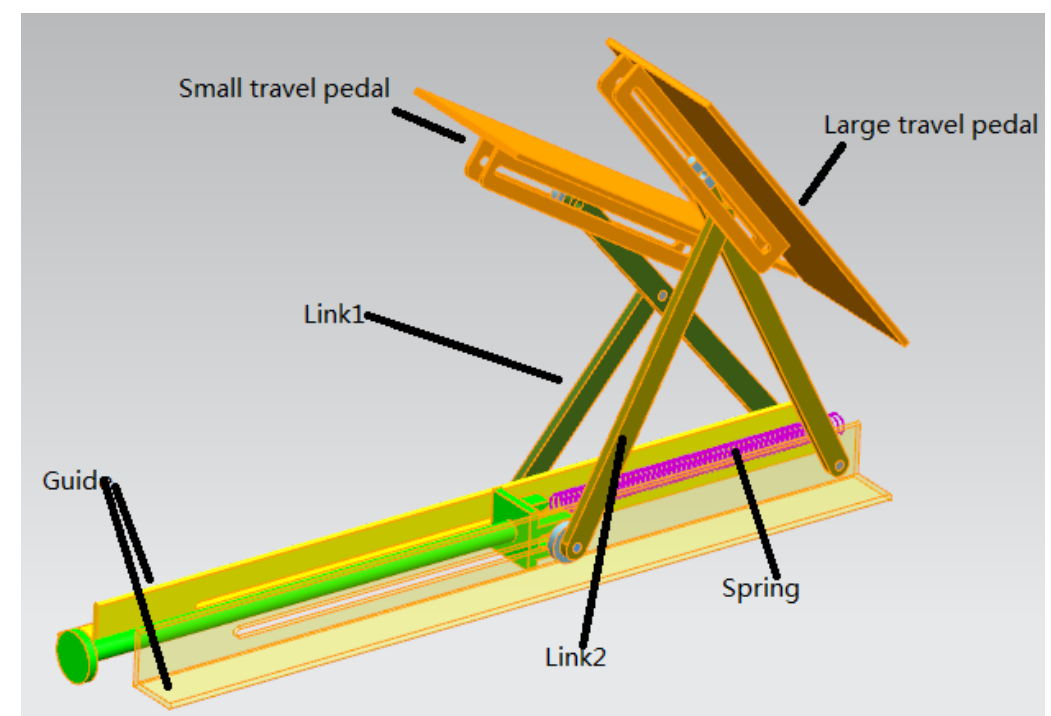

Figure 2 simplified model of new type pump

\section{The Determination of New Type Pump’s Structure Parameters}

In Figure 1 and Figure 2, pump plunger always run out as the action of the spring when not used, slide displacement of link's bottom point of junction is equivalent of piston displacement. according to the pressure range of bicycle tire (400 800kpa), electric bicycle tire $(2.2 \sim 2.5 \mathrm{MPa})$ and less inflation times, the tire inflated to pressure rating need 40 times using current portable inflator products. So selecting the appropriate pump: inner diameter of outer sleeve was $45 \mathrm{~mm}$, thickness was $1 \mathrm{~mm}$, the effective pumping trip maximum was $280 \mathrm{~mm}$ (considering the force of the spring and plunger resistance, could not achieve the maximum inflation stroke). Considering the leg lift height and foot comfort, pedal length, height were tentatively identified as $270 \mathrm{~mm}, 220 \mathrm{~mm}$, pedal tilt angle was 45 degrees.

After confirming the design scheme of new type pumping mechanism, the next task determined the basic structural parameters of the pump, including 1, 2, which bar 1 was set as the length of the rod crank length. Through the relationship between stroke and pressure, these parameters were quickly determined.

Establish the Mathematical Model of the Pneumatic Mechanism. In order to meet the inflation stability, conveniently, the guide was set on the pedal at the bottom, and connecting with bar 1. Taking lower hinge point of the crank as origin $\mathrm{O}$, the horizontal direction as $\mathrm{X}$ axis, vertical direction as $\mathrm{Y}$ axis, the coordinate system was set up as shown in Figure 3. The length of bar 1 set to be a as the crank, which the angle with the horizontal direction set to be $\alpha$; The length of bar 2 set to $b e \mathrm{~b}$ as the crank, which the angle with the horizontal direction set to be $\beta$. The coordinate system value of link sliding point set to be $(x, y)$, since link sliding point move along the horizontal direction, the coordinate system value of link sliding point is $(\mathrm{x}, 0)$ actually, then the following formula was obtained:

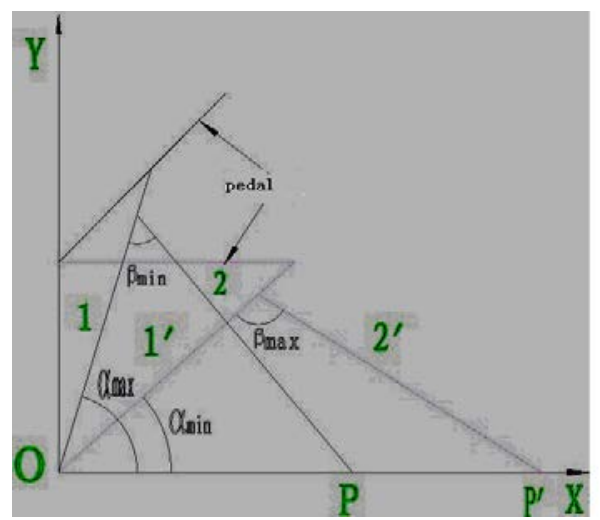

Figure 3 mathematical model of the pneumatic mechanism 


$$
\begin{gathered}
a \cos \alpha+b \cos (180-\alpha-\beta)=x \\
a \sin \alpha=b \sin (180-\alpha-\beta) \\
0<\left[a \cos \alpha_{\text {min }}+b \cos \left(180-\alpha_{\text {min }}-\beta_{\text {max }}\right)\right]-\left[a \cos \alpha_{\text {max }}+b \cos \left(180-\alpha_{\text {max }}-\beta_{\text {min }}\right)\right] \leq 280 \\
0<a \sin \alpha<l \sin 45^{\circ}
\end{gathered}
$$

Where $0^{\circ} \leq \alpha<90^{\circ}, 0^{\circ}<\beta<180^{\circ}, 0<\mathrm{a}<200 \leq \mathrm{b}, \quad$ as the pedal's length.

The Calculation of Filling Quantity. The inner diameter of outer sleeve and thickness were known. Thus filling quantity was calculated in each inflation travel:

$$
Q=\pi \times\left(\frac{45}{2} \times 10^{-3}\right)^{2} \times x \times 10^{-3}
$$

As the common bicycle inner tube size, the average size was about $660 \mathrm{~mm}$ diameter、 2 inches width, full of gas storage capacity was calculated:

$$
Q_{c q}=4.2 \times 10^{-3} \mathrm{~m}^{3}
$$

Inflated number was calculated:

$$
n=\frac{Q_{c q}}{Q}=\frac{4.2 \times 10^{-3}}{\pi \times\left(\frac{45}{2} \times 10^{-3}\right)^{2} \times x \times 10^{-3}}=\frac{8.3 \times 10^{3}}{\pi[a \cos \alpha+b \cos (180-\alpha-\beta)]}
$$

The Optimization of the Organization. The size of the mechanism was optimized mainly from the aeration quantity analysis and kinematic analysis, with the least number of completes the inflation, primary factors affecting the aeration quantity were analyzes : P's displacement PP'. From the mathematical point of view, to solve the optimization problem should first establish the mathematical model and solve by using corresponding optimization method. The model of pneumatic mechanism was established as shown Figure 4. According to the $\mathrm{P}$ trajectories, using software UG and MATLAB, the basic parameters value of structure were confirmed.

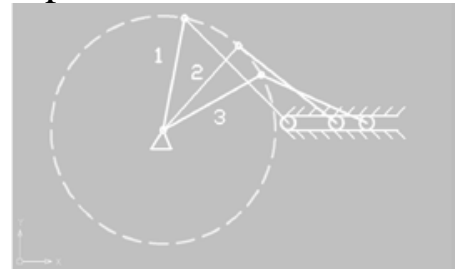

Figure 4 model of pneumatic mechanism

Length of bar 1 rod was $333.02 \mathrm{~mm}$, then as the crank the length was optimized $281.74 \mathrm{~mm}$; length of bar 2 was $352.84 \mathrm{~mm}$, and the point P's displacement was $200 \mathrm{~mm}$.through calculation the least times of inflation was13.2, rounding as14 times.

Shown in Figure 5 and Figure 6, the vertical displacement of hinge point between bar 1 and 2 was $83.09 \mathrm{~mm}$, the horizontal displacement was $128.42 \mathrm{~mm}$, Mechanism motion did not produce interference.

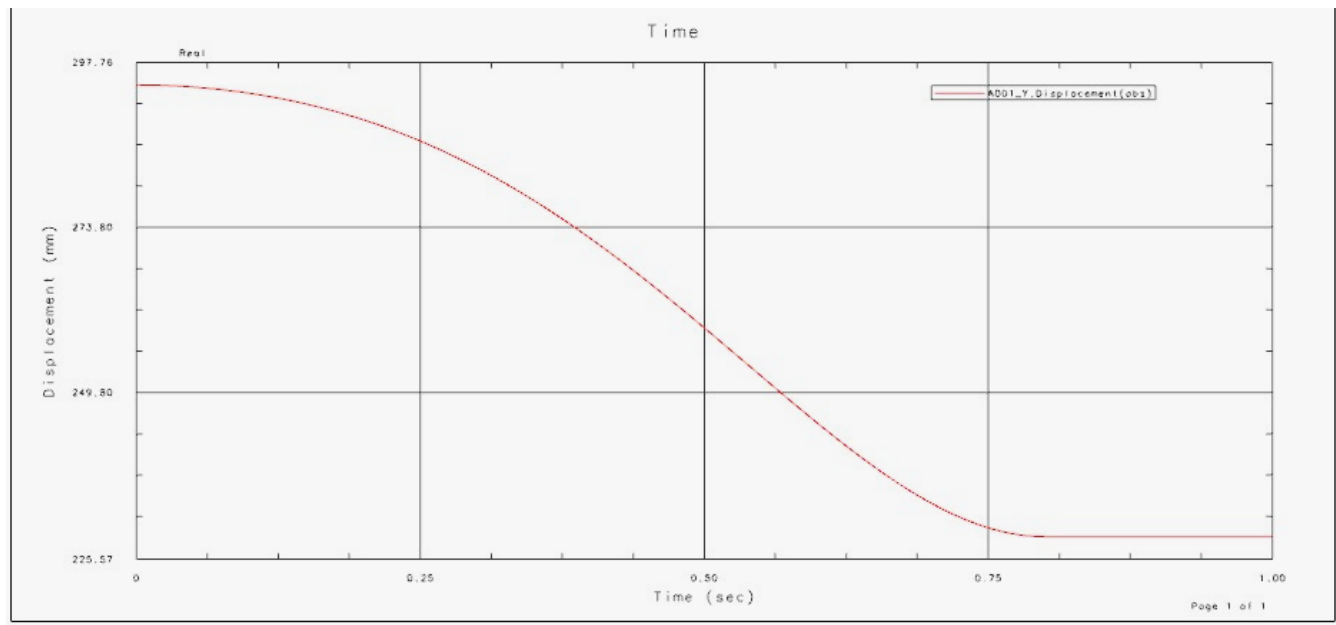

Figure 5 the vertical displacement of hinge point between bar 1 and 2 


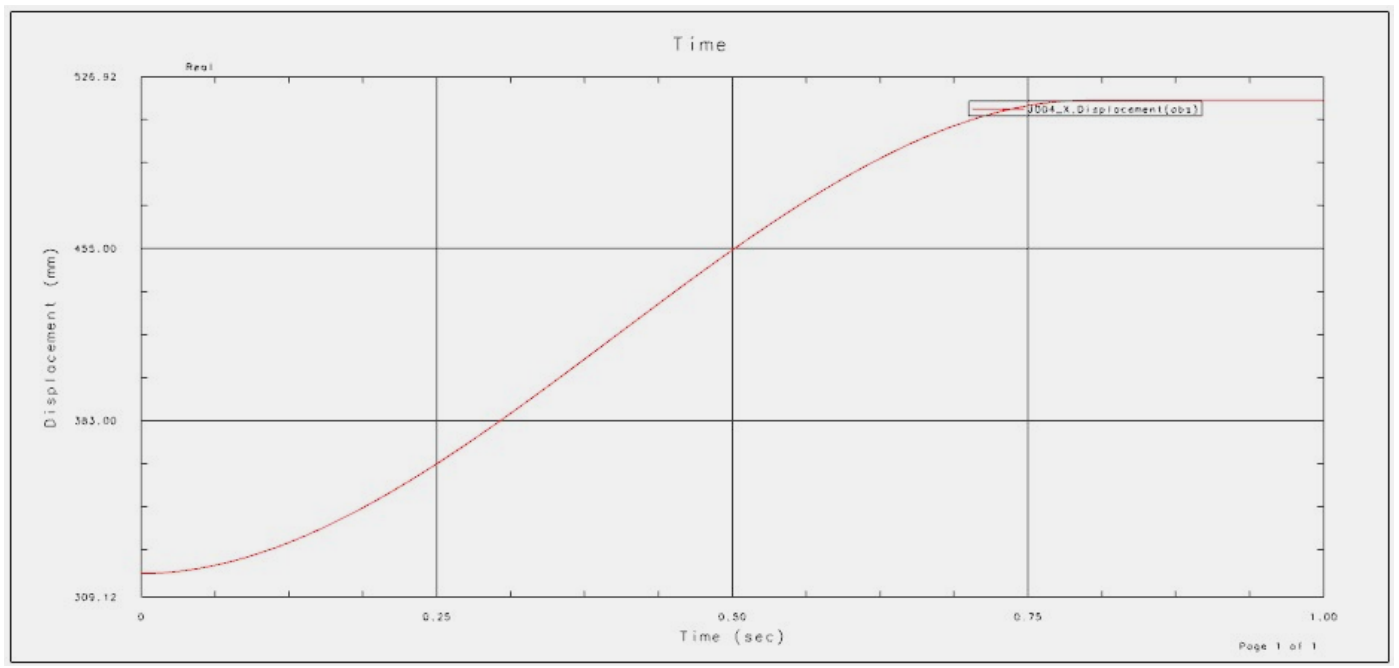

Figure 6 horizontal displacement of hinge point between bar 1 and 2

\section{Design of Overload Protection Device}

The tee-joint valve was set in the pipeline, which was connected with a pressure gauge, another connected tube. Tube pressure value was read from pressure gauge. When the valve reaches 1 , the safety valve 2 close; When the valve reaches 2 , the safety valve 1 close. When the pipeline pressure reaches the rated pressure value, the safety valve open to overload protect. In general, the safety valve 1 was set as bicycle safety valve; safety valve 2 was set as electric protection valve.

\section{Conclusion}

This paper presented a new design method for the pedal inflator charging mechanism scheme, abandoning the traditional method of trial and error for generating mechanism type synthesis. Applying the mechanism type synthesis, the crank slider mechanism was determined as the inflation mechanism. Establishing the mathematical model of inflation mechanism, and determining the objective function and constraint conditions, then the structure parameters of the inflation mechanism was quickly determined applying the software of MATLAB and UG. The optimization design results was test, verify through the prototype as shown Figure 7, achieving the aim of the design.

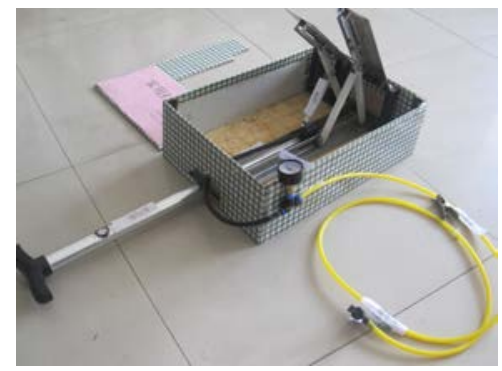

Figure 7 prototype

\section{Reference}

[1] Tingli Yang. Topology Structure Design of Robot Mechanisms [M]. Beijing: Machinery Industry Press,2004.10 13

[2] Chao Wang, Hui Si. Design of Bicycle Lock Type Pump [J]. Forestry Machinery \& Woodworking Equipment. 2012,12,Vo1 40 No.6

[3] Wei Guo, Jiajun Ren. The Optimal Design of Crank Rocker Movement Rules[J]. Mechanical Management And Development. 2006, 4, P35-36. 
[4] Wenjuan Wang. Optimal Design of Planar Linkage Based on MATLAB Optimization Toolbox[J]. Light Industry Machinery. 2006, 12, Vol.24, NO.4, P76-78.

[5] Jie An, Yuzahng Zou. UG post processing technique[M].Beijing: Tsinghua University press. 2003. 